\title{
Abandono de la terapia nutricional y pérdida de peso en pacientes bariátricos: Cohorte retrospectiva en Lima, Perú
}

\section{Monica Giacchetti-Vega ${ }^{a}, *$, Pamela Baquerizo-vonBerswordts ${ }^{a}$, Camila Carbone-Moane ${ }^{a}$, Antonio Bernabé-Ortiz ${ }^{a}$}

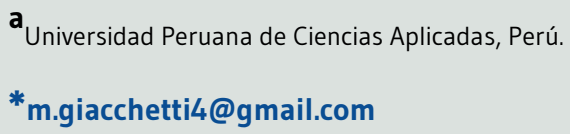

Recibido el 1 de agosto de 2016; aceptado el 2 de enero de 2017.

\section{PALABRAS CLAVE}

Cirugía bariátrica;

Obesidad mórbida;

Terapia dietética;

Abandono de pacientes;

Gestión de la atención al paciente.
Abandono de la terapia nutricional y pérdida de peso en pacientes bariátricos: Cohorte retrospectiva en Lima, Perú

\section{RESUMEN}

Introducción: El objetivo de este trabajo fue determinar la proporción de pacientes que cumplen criterios internacionales de gastrectomía en manga, así como estimar la asociación entre porcentaje de pérdida de peso con tasa de abandono del tratamiento nutricional.

Material y Métodos: Cohorte retrospectiva realizada con historias clínicas de pacientes con gastrectomía en manga entre 2008-2014 en una clínica privada. Hubo dos variables resultado de interés: los criterios de inclusión para cirugía bariátrica según criterios internacionales (IMC $\geq 40 \mathrm{~kg} / \mathrm{m}^{2}$ $0 \geq 35 \mathrm{~kg} / \mathrm{m}^{2}$ con comorbilidades) y el abandono antes o durante (inasistencia $\geq 60$ días desde la consulta previa sin recibir alta [IMC $<25]$ ) del tratamiento nutricional. La exposición fue el porcentaje de peso perdido en las primeras cinco semanas después de la cirugía $(<10 \%$ vs. $\geq 10 \%$ ). Se utilizaron modelos de regresión de Poisson. Se calcularon riesgos relativos e intervalos de confianza al 95\% (IC95\%).

Resultados: La muestra incluyó 423 historias clínicas, 294 (69,5\%) mujeres, edad media 39,2 $( \pm 12,3)$ años. $117(27,7 \%)$ cumplieron requisitos para cirugía y 48 de esos 117 (41\%) abandonaron el tratamiento nutricional antes de iniciarlo. Los pacientes que perdieron $\geq 10 \%$ de peso en el primer mes tuvieron un $90 \%$ menos de riesgo de abandonar la terapia nutricional que los que perdieron $<10 \%$ del peso (RR=0,10; IC95\%:0,01-0,68), ajustando por sexo y edad.

Conclusiones: La mayoría de pacientes bariátricos no cumple los requisitos internacionales. Una mayor pérdida de peso en el primer mes se asoció a un menor abandono del tratamiento nutricional. 
Diet and weight loss therapy dropout in bariatric patients: Retrospective cohort in Lima, Peru

\section{KEYWORDS}

Bariatric Surgery;

Obesity, Morbid;

Diet Therapy;

Patient Dropouts;

Patient Care

Management.

\section{ABSTRACT}

Introduction: The aims of this study were to determine the proportion of patients who meet international recommendations for bariatric surgery and to assess the association between weight loss and dropout rate during diet therapy.

Material and Methods: Retrospective cohort study performed using data from clinical records of patients who underwent sleeve gastrectomy in a private clinic of Lima, Peru. Two outcomes were evaluated, eligibility for bariatric surgery according to international recommendations (BMl $\geq 40 \mathrm{~kg} / \mathrm{m}^{2}$ or $\geq 35 \mathrm{~kg} / \mathrm{m}^{2}$ with comorbidities) and abandonment before and after starting nutritional management (no nutritional assessment after 60 days from the previous visit without achieving ideal BMI). The exposure was the percentage of weight lost in the first five weeks after surgery split into $<10 \%$ and $\geq 10 \%$. Poisson regression was used and relative risks and $95 \%$ confidence intervals $(95 \% \mathrm{Cl})$ were calculated.

Results: Data from 423 medical records of bariatric patients, mean age 39.2 years $( \pm 12.3), 294$ (69.5\%) women, were analyzed. Only 117 (27.7\%) patients met international recommendations for bariatric surgery. Before starting nutritional management, 48/117 (41\%) abandoned treatment, whereas an additional 29/69 (42.0\%) abandoned before six months of nutritional management. Patient losing $\geq 10 \%$ of weight in the first month had $90 \%$ lower risk ( $R R=0.10 ; 95 \% \mathrm{Cl}: 0.01-0.68$ ) of nutritional therapy dropout compared to those who lost $<10 \%$ of weight, controlling for sex and age.

Conclusions: A great proportion of patients undergoing bariatric surgery did not meet the international criteria. A greater loss of weight during the first month was associated a lower dropout rate during nutritional therapy.

\section{CITA}

Giacchetti-Vega M, Baquerizo-vonBerswordts P, Carbone-Moane C, Bernabé-Ortiz A. Abandono de la terapia nutricional y pérdida de peso en pacientes bariátricos: Cohorte retrospectiva en Lima, Perú. Rev Esp Nutr Hum Diet. 2017; 21(1): 18-28. doi: 10.14306/renhyd.21.1.271

\section{INTRODUCCIÓN}

En el 2014, 266 millones de varones y 375 millones de mujeres presentaron obesidad en el mundo y se estima que para 2025 la prevalencia mundial alcance $18 \%$ y $21 \%$ en varones y mujeres, respectivamente ${ }^{1}$. En Lima Metropolitana, el $11,4 \%$ de los adultos entre $20-29$ años y el $23,5 \%$ de los adultos entre 30-59 años presentó obesidad en el 2010². La obesidad es considerada un factor de riesgo importante para diabetes, enfermedades cardiovasculares, cáncer y muerte prematura ${ }^{3}$. La dieta y el ejercicio son considerados un tratamiento convencional para la pérdida de peso, pero se considera a la cirugía bariátrica como un tratamiento más efectivo para tratar la obesidad, disminuir factores de riesgo y mejorar el estilo de vida en personas con obesidad severa $a^{4}$.

La implementación de cirugía bariátrica como estrategia para reducir la obesidad requiere un protocolo apropiado. En Estados Unidos y Europa se han establecido protocolos a nivel nacional para cirugía bariátrica, en los cuales se resaltan las indicaciones para elegir al candidato a ser sometido a cirugía bariátrica y se enfatiza el monitoreo del paciente por un equipo multidisciplinario (médico, psicólogo, nutricionista) antes y después de la cirugía ${ }^{5-7}$. Entre los países 
latinoamericanos, Brasil y Chile han desarrollado protocolos para cirugía bariátrica ${ }^{8,9}$. Chile enfatiza el tratamiento integral de la obesidad teniendo en cuenta parámetros psicológicos para los criterios de inclusión del paciente y cuenta con una nutricionista en el equipo clínico pre- y poscirugía.

En Perú son varios los centros tanto públicos como privados que han llevado a cabo la cirugía bariátrica por años ${ }^{10}$, pero no se cuenta con un protocolo estandarizado a nivel nacional sobre el uso de esta cirugía en el tratamiento de la obesidad. En cambio, las indicaciones y manejo varían según los hospitales o clínicas que la realizan. En la ausencia de un protocolo estandarizado a nivel nacional, el tratamiento nutricional no ha sido establecido y la cantidad de pacientes que lo comienzan, terminan o abandonan no ha sido cuantificada.

El tratamiento nutricional es importante en el seguimiento de pacientes bariátricos y puede evitar una re-ganancia de peso, así como complicaciones descritas en la literatura ${ }^{11,12}$. También se ha descrito una alta incidencia en el abandono del tratamiento nutricional poscirugía, indicando los siguientes factores de riesgo: mientras disminuye la edad o el índice de masa corporal, aumenta la probabilidad de abandono ${ }^{13}$. Pero no se han localizado reportes que evalúen el impacto de la pérdida de peso en el abandono del tratamiento nutricional del paciente bariátrico.

La pérdida de peso de un paciente a través de intervenciones dietéticas cuenta con diversos predictores. La pérdida de peso disminuye ante ciertos factores, como los psicológicos (estrés y depresión), malos hábitos alimenticios, poca actividad física y pertenecer al género femenino ${ }^{14}$. Sin embargo, la adicción a la comida, definida como mayor tiempo y cantidad de ingesta, síntomas físicos ante abstinencia e intentos fallidos de reducir las comidas, no disminuyen la pérdida de peso ni afectan el abandono del tratamiento dietético para la obesidad ${ }^{15}$. Se ha postulado evaluar la expectativa de pérdida de peso como predictor de pérdida de peso y factor de riesgo del abandono del tratamiento nutricional, pero un metaanálisis de 13 estudios concluyó que varía con el tiempo y los hallazgos no fueron claros ${ }^{16}$.

Debido a que la obesidad es un problema de salud pública y la cirugía bariátrica es un tratamiento en casos severos, se deben conocer las características de la población y tasa de abandono del tratamiento nutricional para poder implementar estrategias de manejo adecuadas. La literatura identifica distintos factores de riesgo de abandono como el índice de masa corporal (IMC) y por experiencia se ha observado que la pérdida de peso influye en la adherencia a un tratamiento dietético. Por ende, el presente estudio busca estimar la asociación entre el porcentaje de pérdida de peso en el primer, tercer y sexto mes del tratamiento nutricional con la tasa de abandono del mismo; así como, determinar la proporción de pacientes que cumplen los criterios internacionales de gastrectomía en manga.

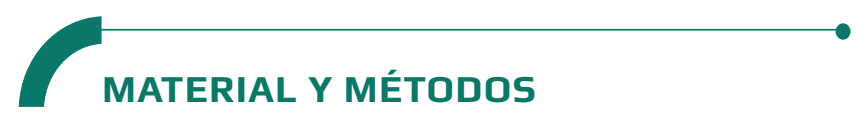

Estudio de tipo cohorte retrospectivo involucrando datos de las historias clínicas de pacientes de una clínica privada de Lima que fueron operados de cirugía bariátrica de tipo gastrectomía en manga desde el año 2008 hasta marzo 2014. El estudio incluyó 478 historias clínicas.

Se realizó un censo de los pacientes bariátricos de la clínica privada donde se realizó el estudio. Asumiendo un nivel de confianza del 95\% (IC95\%) y una potencia del $80 \%$, se requerían como mínimo 288 participantes (144 participantes en cada grupo de acuerdo a exposición) para detectar una diferencia de $16 \%$ en las tasas de abandono, es decir, $46 \%^{17}$ de abandono en aquellos que pierden menos del $10 \%$ de su peso, comparados con $30 \%{ }^{18}$ de abandono en aquellos que pierden más del $10 \%$.

Dos variables resultado fueron evaluadas: los criterios de inclusión para la cirugía bariátrica, definida según las guías americanas y europeas $s^{6,7}$, es decir, aquel paciente que tiene un $\mathrm{IMC} \geq 40 \mathrm{~kg} / \mathrm{m}^{2} \mathrm{o} \geq 35 \mathrm{~kg} / \mathrm{m}^{2}$ con comorbilidades como hipertensión y diabetes; y el abandono del tratamiento nutricional, entendido como la consulta y seguimiento llevado a cabo exclusivamente por la nutricionista, profesional que forma parte del equipo multidisciplinario según indicaciones internacionales ${ }^{6,7,9}$. El abandono fue definido en dos momentos. El primero fue el abandono pretratamiento nutricional, en el que el paciente no acudió a ninguna cita con la nutricionista posterior a la cirugía bariátrica. La segunda fue el abandono del tratamiento nutricional evaluado desde la primera cita con la nutricionista. Se definió como haber asistido a una sola consulta nutricional y, a partir del segundo mes de seguimiento, se consideró cuando hubo inasistencia por un espacio de tiempo >60 días desde la consulta previa sin haber sido dado de alta (IMC $\left.<25 \mathrm{~kg} / \mathrm{m}^{2}\right)$.

La variable de exposición de interés fue el porcentaje de peso perdido obtenido con la siguiente fórmula ${ }^{19}$ :

$$
\text { Porcentaje de peso perdido }=\frac{P_{0}-P_{1}}{P_{0}} \cdot 100
$$

En la cual $P_{0}$ fue el peso en el momento en que se realizó la cirugía bariátrica y $P_{1}$ fue el peso cinco semanas después de 
la cirugía; es decir, cuando debe comenzar el tratamiento nutricional. Para el análisis se utilizó como punto de corte $10 \%^{17,18}$ y se definieron dos categorías: $<10 \%$ y $\geq 10 \%$.

Las variables sociodemográficas consideradas en el análisis fueron: sexo, edad (en años, <30; 30-39; 40-49; 50-59; $\geq 60)$, IMC (<30; 30-34,9; 35-39,9; $\geq 40)$, consumo de alcohol, tabaquismo y uso de drogas. Las variables de antecedentes personales fueron: diabetes, hipertensión e hipotiroidismo.

A pesar de no existir un protocolo estandarizado para cirugía bariátrica, los pacientes asistieron a una evaluación clínica, medición de talla y peso, así como exámenes prequirúrgicos. Los pacientes fueron pesados nuevamente en el día de la cirugía y una semana después.

Cinco semanas después de la cirugía el paciente tiene su primera consulta con la nutricionista y se da inicio al tratamiento nutricional, el cual es llevado a cabo solamente por la nutricionista. Durante el primer mes de tratamiento nutricional se acude 2 veces al mes y durante los siguientes se asiste de manera mensual. Se realiza el seguimiento del peso en cada consulta hasta el alta del tratamiento (alcanzar IMC $<25 \mathrm{~kg} / \mathrm{m}^{2}$ ). Para fines de este estudio, se usó la información del primer, tercero y sexto mes subsiguiente a la primera cita nutricional.

Para la recolección de datos se diseñó una plantilla especial. Primero, se revisaron las historias de la nutricionista para obtener las fechas y pesos de las consultas. Posteriormente, se revisaron las historias clínicas del médico para recopilar datos sobre los antecedentes personales, talla y fecha de cirugía.

Se llevó a cabo una doble digitación utilizando el programa Excel para Windows y los datos fueron luego transferidos a STATA 13 para Windows (STATA CORP, College Station, TX, US) para el análisis.

Inicialmente, la descripción de la población de estudio fue realizada usando medias y desviación estándar (DE) para variables numéricas, mientras que frecuencias y proporciones se usaron para las variables categóricas. Para la comparación de variables categóricas se utilizó la prueba $\chi^{2}$ o la prueba exacta de Fisher según correspondiera.

Se calculó la proporción de pacientes que cumplían con los requisitos para ser operado y luego, se calcularon las tasas de abandono antes del tratamiento nutricional y posterior a este en sólo aquellos que cumplían los requisitos. Para evaluar los factores asociados según el tipo de abandono, se usaron modelos de regresión de Poisson con varianza robusta y se calcularon riesgos relativos y sus respectivos IC95\%. Finalmente, en análisis aparte, se evaluó si el porcentaje de peso perdido estuvo asociado a abandono posterior al mes, tres meses, y seis meses posteriores al inicio del tratamiento nutricional, controlando por sexo y edad.

Dado que la institución evaluada no presentaba comité de ética, el proyecto de investigación fue presentado, revisado y aprobado por el Comité de Ética en Investigación de la Universidad Peruana de Ciencias Aplicadas, Lima, Perú.

No hubo consentimiento informado debido a que no se trató directamente con los pacientes. Se garantizó el anonimato y confidencialidad de los datos registrados de las historias clínicas nutricionales.

\section{RESULTADOS}

Se obtuvieron las historias clínicas de 478 pacientes sometidos a cirugía bariátrica en el período de estudio. De éstas, 55 no cumplieron con los criterios de inclusión: 22 fueron operados con una técnica bariátrica distinta a la gastrectomía en manga laparoscópica y 33 tuvieron los datos incompletos en la historia clínica. Por ello, sólo se analizaron los datos de 423 pacientes bariátricos (Figura 1). La edad al momento de la cirugía fue 39,2 ( $\pm 12,3)$ y $294(69,5 \%)$ fueron mujeres.

Figura 1. Diagrama de flujo de los participantes del estudio.

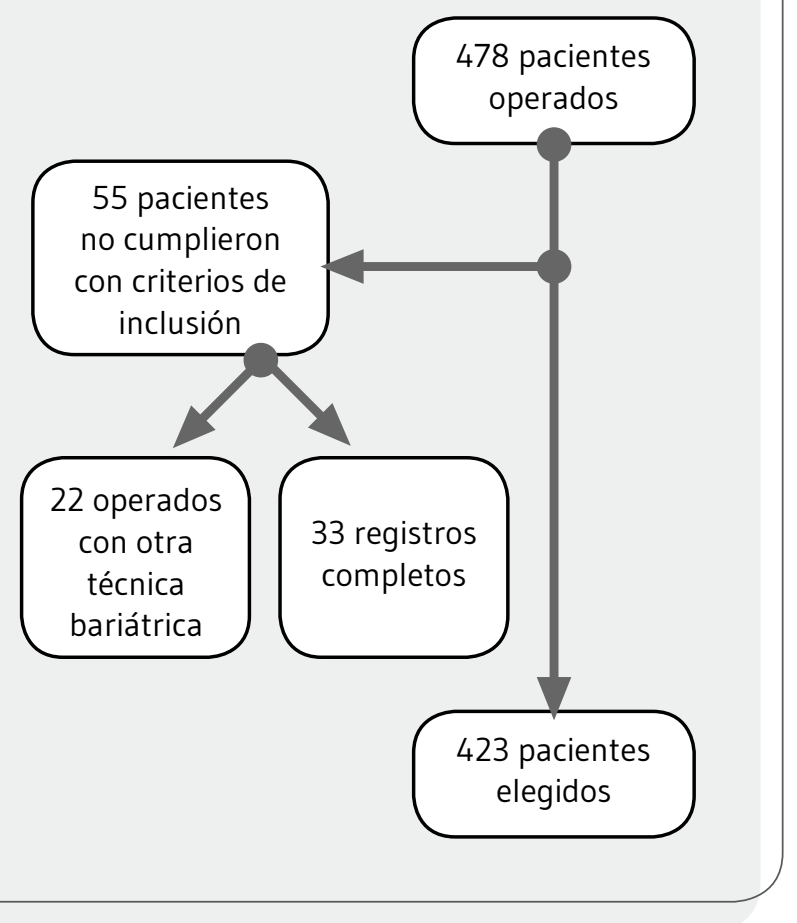


Sólo $117(27,7 \%)$ de los pacientes sometidos a cirugía bariátrica cumplieron con los criterios establecidos internacionalmente para ser sometidos a cirugía bariátrica y fueron estos los que fueron analizados posteriormente. El IMC promedio de esta población al momento de la cirugía fue $44,5 \mathrm{~kg} / \mathrm{m}^{2}$ $( \pm 5,2)$.Las características de la población según los criterios de inclusión se muestran en la Tabla 1. Casi dos tercios del grupo no elegible tuvo un IMC menor a $35 \mathrm{~kg} / \mathrm{m}^{2}$ y una mayor proporción de mujeres no fue elegible según criterios internacionales $(p<0,001)$. Adicionalmente, hubo diferencias estadísticamente significativas en el reporte de consumo de alcohol $(p=0,02)$, y en antecedentes de hipertensión arterial $(p<0,001)$, diabetes mellitus $(p<0,001)$ e hipotiroidismo $(p=0,04)$.

Tabla 1. Características de la población de estudio según criterios de inclusión para cirugía bariátrica.

\begin{tabular}{|c|c|c|c|}
\hline & $\begin{array}{c}\text { Cumplen criterios } \\
\text { de inclusión } \\
n(\%)\end{array}$ & $\begin{array}{c}\text { No cumplen criterios } \\
\text { de inclusión } \\
n(\%)\end{array}$ & $p$-valor \\
\hline \multicolumn{4}{|l|}{ Sexo } \\
\hline Femenino & $55(47,0)$ & $239(78,1)$ & $<0,001$ \\
\hline Masculino & $62(53,0)$ & $62(21,9)$ & \\
\hline \multicolumn{4}{|l|}{ Edad* (años) } \\
\hline$<30$ & $18(15,4)$ & $84(27,5)$ & 0,11 \\
\hline $30-39$ & $37(31,6)$ & $73(23,9)$ & \\
\hline $40-49$ & $33(28,2)$ & $82(26,9)$ & \\
\hline $50-59$ & $22(18,8)$ & $48(15,7)$ & \\
\hline$>60$ & $7(6,0)$ & $18(5,9)$ & \\
\hline \multicolumn{4}{|l|}{ IMC $\left(\mathrm{kg} / \mathrm{m}^{2}\right)$} \\
\hline$<30$ & $0(0)$ & $45(14,7)$ & $<0,001$ \\
\hline $30-34,9$ & $0(0)$ & $151(49,4)$ & \\
\hline $35-39,9$ & $10(8,6)$ & $110(36,0)$ & \\
\hline$\geq 40$ & $107(91,5)$ & $0(0)$ & \\
\hline \multicolumn{4}{|c|}{ Consumo de alcohol } \\
\hline No & $43(36,8)$ & $76(24,8)$ & 0,015 \\
\hline Sí & $74(63,3)$ & $230(75,2)$ & \\
\hline \multicolumn{4}{|l|}{ Tabaquismo } \\
\hline No & $79(67,5)$ & $209(68,3)$ & 0,88 \\
\hline Sí & $38(32,5)$ & $97(31,7)$ & \\
\hline \multicolumn{4}{|l|}{ Uso de drogas } \\
\hline No & $117(100)$ & $301(98,4)$ & 0,16 \\
\hline Sí & $0(0)$ & $5(1,6)$ & \\
\hline
\end{tabular}

Antecedentes personales

\begin{tabular}{l|c|c|c}
\hline Hipertensión arterial & & & \\
\hline No & $71(60,7)$ & $253(82,7)$ & $<0,001$ \\
Sí & $46(39,3)$ & $53(17,3)$ & \\
\hline Diabetes mellitus 2 & & & \\
\hline No & $98(83,8)$ & $290(94,8)$ & $<0,001$ \\
Sí & $19(16,2)$ & $16(5,2)$ & \\
\hline Hipotiroidismo & $109(93,2)$ & $262(85,6)$ & 0,035 \\
\hline No & $8(6,8)$ & $44(14,4)$ & \\
Sí & & & \\
\hline
\end{tabular}

*Edad tiene una población diferente a la total $(n=422)$ debido a fichas incompletas.

IMC: Índice de Masa Corporal; $\mathbf{n}$ : muestra. 
Entre quienes cumplieron los criterios de inclusión para la cirugía, el 41,0\% (IC95\%:32,0-50,1\%) nunca asistió al tratamiento nutricional. Ninguno de los factores sociodemográficos o clínicos evaluados estuvo asociado a este tipo de abandono (Tabla 2).

De aquellos pacientes que acudieron al tratamiento nutricional, el 15,9\% abandonaron en el primer mes de haber iniciado el tratamiento, el 21,7\% abandonaron a los 3 meses y 42,0\% abandonaron en los primeros 6 meses. El 20,3\% continuó el tratamiento nutricional por más de 6 meses. Las características de la población de estudio según el momento de abandono se muestran en la Tabla 3. Ningún factor estuvo asociado al abandono del tratamiento nutricional al mes, 3 meses o 6 meses de seguimiento (Tabla 4).

Controlando por sexo y edad, los que perdieron más del $10 \%$ de su peso en las primeras cinco semanas poscirugía tuvieron $90 \%$ menos probabilidad de abandonar el tratamiento nutricional en el primer mes de tratamiento $(R R=0,10$; IC95\%:0,01-0,68) respecto a los que perdieron $<10 \%$ del peso. La pérdida de peso, sin embargo, no estuvo asociada al abandono durante el tercer y sexto mes de tratamiento (Tabla 5).

\section{$\longrightarrow$ \\ DISCUSIÓN}

De acuerdo a los resultados del presente estudio, cerca del $75 \%$ de los pacientes sometidos a cirugía bariátrica no cumplieron los requisitos internacionales para ser operados. Además, 4 de cada 10 pacientes sometidos a cirugía bariátrica que cumplieron los criterios de inclusión abandonaron el tratamiento y seguimiento antes de acudir al tratamiento nutricional, y de los que sí acudieron, el 79,7\% no llegaron a los seis meses de seguimiento. Finalmente, después de controlar por edad y sexo, aquellos pacientes que perdieron más del $10 \%$ tuvieron menor probabilidad de abandonar el tratamiento nutricional en el primer mes, comparado con los que perdieron $<10 \%$ de peso. Sin embargo, el porcentaje de pérdida de peso no estuvo asociado al abandono en el tercer o sexto mes de seguimiento.

Tabla 2. Comparación de acuerdo a abandono pretratamiento nutricional ( $n=117)$.

\begin{tabular}{|c|c|c|}
\hline & Bivariado & Multivariado* \\
\hline & RR (IC95\%) & RR (IC95\%) \\
\hline \multicolumn{3}{|l|}{ Sexo } \\
\hline Femenino & Referencia & Referencia \\
\hline Masculino & $1,48(0,93-2,34)$ & $1,55(0,96-2,53)$ \\
\hline \multicolumn{3}{|l|}{ Edad (años) } \\
\hline$<30$ & Referencia & Referencia \\
\hline $30-39$ & $0,61(0,29-1,29)$ & $0,66(0,31-1,42)$ \\
\hline $40-49$ & $1,09(0,58-2,04)$ & $1,01(0,53-1,91)$ \\
\hline $50-59$ & $1,13(0,58-2,19)$ & $1,25(0,60-2,64)$ \\
\hline$>60$ & $0,96(0,35-2,63)$ & $0,99(0,37-2,65)$ \\
\hline \multicolumn{3}{|l|}{ IMC $\left(\mathrm{kg} / \mathrm{m}^{2}\right)$} \\
\hline $35-39,9$ & Referencia & Referencia \\
\hline$\geq 40$ & $0,65(0,37-1,15)$ & $0,25(0,04-1,70)$ \\
\hline \multicolumn{3}{|l|}{ Estilos de vida } \\
\hline Consumo de alcohol & $0,69(0,45-1,05)$ & $0,67(0,44-1,04)$ \\
\hline Tabaquismo & $1,14(0,73-1,79)$ & $1,35(0,88-2,08)$ \\
\hline \multicolumn{3}{|l|}{ Antecedentes personales } \\
\hline Hipertensión arterial & $1,20(0,78-1,85)$ & $1,13(0,64-1,99)$ \\
\hline Diabetes mellitus 2 & $0,88(0,47-1,66)$ & $0,25(0,03-1,88)$ \\
\hline Hipotiroidismo & $1,24(0,60-2,58)$ & $1,82(0,89-3,69)$ \\
\hline
\end{tabular}

IMC: Índice de Masa Corporal; RR: Riesgo Relativo; IC95\%: Intervalo de Confianza 95\%. * Modelo presentado está ajustado por todas las variables listadas en la tabla. 
Tabla 3. Comparación de acuerdo al abandono del tratamiento nutricional.

\begin{tabular}{|c|c|c|c|c|c|c|}
\hline \multirow[b]{2}{*}{$\begin{array}{l}\text { Abandono del tratamiento } \\
\text { nutricional }\end{array}$} & \multicolumn{2}{|c|}{1 MES } & \multicolumn{2}{|c|}{3 MESES } & \multicolumn{2}{|c|}{6 MESES } \\
\hline & $\begin{array}{l}\text { No (58) } \\
\text { n (\%) }\end{array}$ & $\begin{array}{l}\text { Sí (11) } \\
\text { n (\%) }\end{array}$ & $\begin{array}{l}\text { No (54) } \\
\text { n (\%) }\end{array}$ & $\begin{array}{l}\text { Sí (15) } \\
\text { n (\%) }\end{array}$ & $\begin{array}{l}\text { No (40) } \\
n(\%)\end{array}$ & $\begin{array}{l}\text { Sí (29) } \\
\text { n (\%) }\end{array}$ \\
\hline \multicolumn{7}{|l|}{ Sexo } \\
\hline Femenino & $33(89,2)$ & $4(10,8)$ & $29(78,4)$ & $8(21,6)$ & $25(67,6)$ & $12(32,4)$ \\
\hline Masculino & $25(78,1)$ & $7(21,9)$ & $25(78,1)$ & $7(21,9)$ & $15(46,9)$ & $17(53,1)$ \\
\hline \multicolumn{7}{|l|}{ Edad (años) } \\
\hline$<30$ & $9(90)$ & $1(10)$ & $6(60,0)$ & $4(40,0)$ & $7(70,0)$ & $3(30,0)$ \\
\hline $30-39$ & $20(74,1)$ & $7(25,9)$ & $21(77,8)$ & $6(22,2)$ & $12(44,4)$ & $15(55,7)$ \\
\hline $40-49$ & $16(94,1)$ & $1(5,9)$ & $14(82,4)$ & $3(17,7)$ & $11(64,7)$ & $6(35,3)$ \\
\hline $50-59$ & $9(81,8)$ & $2(18,2)$ & $10(90,9)$ & $1(9,1)$ & $8(72,7)$ & $3(27,3)$ \\
\hline$>60$ & $4(100)$ & $0(0)$ & $3(75,0)$ & $1(25,0)$ & $2(50,0)$ & $2(50,0)$ \\
\hline \multicolumn{7}{|l|}{ IMC (kg/m²) } \\
\hline $35-39,9$ & $3(75,0)$ & $1(25,0)$ & $4(100,0)$ & $0(0)$ & $2(50,0)$ & $2(50,0)$ \\
\hline$\geq 40$ & $55(84,6)$ & $10(15,4)$ & $50(76,9)$ & $15(23,1)$ & $38(58,5)$ & $27(41,5)$ \\
\hline
\end{tabular}

Estilos de vida

\begin{tabular}{c|c|c|c|c|c|c|c|}
\hline $\begin{array}{c}\text { Consumo de alcohol } \\
\text { Sí }\end{array}$ & $38(79,2)$ & $10(20,8)$ & $38(79,2)$ & $10(20,8)$ & $24(50,0)$ & $24(50,0)$ \\
No & $20(95,2)$ & $1(4,8)$ & $16(76,2)$ & $5(23,8)$ & $16(76,2)$ & $5(23,8)$ \\
\hline Tabaquismo & & & & & & \\
\hline Sí & $17(81,0)$ & $4(19,1)$ & $16(76,2)$ & $5(23,8)$ & $11(52,4)$ & $10(47,6)$ \\
No & $41(85,4)$ & $7(14,6)$ & $38(79,2)$ & $10(20,8)$ & $29(60,4)$ & $19(39,6)$ \\
\hline
\end{tabular}

Antecedentes personales

\begin{tabular}{|c|c|c|c|c|c|c|}
\hline $\begin{array}{c}\text { Hipertensión arterial } \\
\text { Sí }\end{array}$ & & & & & \\
No & $20(80,0)$ & $5(20,0)$ & $19(76,0)$ & $6(24,0)$ & $12(48,0)$ & $13(52,0)$ \\
\hline Diabetes mellitus 2 & $38(86,4)$ & $6(13,6)$ & $35(79,6)$ & $9(20,5)$ & $28(63,6)$ & $16(36,4)$ \\
\hline Sí & & & & & & \\
No & $10(83,3)$ & $2(16,7)$ & $11(91,7)$ & $1(8,3)$ & $7(58,3)$ & $5(41,7)$ \\
\hline Hipotiroidismo & $48(84,2)$ & $9(15,8)$ & $43(75,4)$ & $14(24,6)$ & $33(57,9)$ & $24(42,1)$ \\
\hline Sí & & & & & & \\
No & $3(75,0)$ & $1(25,0)$ & $4(100,0)$ & $0(0,0)$ & $2(50,0)$ & $2(50,0)$ \\
& $55(84,6)$ & $10(15,4)$ & $50(76,9)$ & $15(23,1)$ & $38(58,5)$ & $27(41,5)$ \\
\hline
\end{tabular}

IMC: Índice de Masa Corporal; n: muestra.

Una gran proporción de los pacientes bariátricos no cumplió con los criterios internacionales basados en IMC y la presencia de comorbilidades. Se ha establecido a la cirugía bariátrica como parte integral de un manejo para pacientes con obesidad mórbida ${ }^{6}$. Por ello, la falta de un protocolo adecuado en el contexto peruano podría contribuir a las variaciones en indicaciones clínicas y el uso de cirugía bariátrica ${ }^{20}$. En el ámbito público, el Hospital Edgardo Rebagliati Martins cuenta con una "Guía de Práctica ClínicaQuirúrgica. Obesidad Clínica Severa", la cual fue aprobada por la resolución de gerencia central de prestaciones de salud número 77 GCPS de ESSALUD. Sin embargo, el contenido de dicha guía no está disponible para el público. A pesar de un consenso claro en protocolos clínicos, en un estudio involucrando distintos países europeos ${ }^{5}$, los pacientes eran elegibles sin comorbilidades si su IMC se encontraba entre $40-50 \mathrm{~kg} / \mathrm{m}^{2}$ en Dinamarca e Inglaterra, mientras que en el resto de países eran elegibles con comorbilidades y un IMC mayor a $35 \mathrm{~kg} / \mathrm{m}^{2}$. Además, aproximadamente el $5,4 \%$ de la población adulta podría ser elegible para cirugía bariátrica 
Tabla 4. Modelo de regresión bivariado y multivariado para abandono del tratamiento nutricional.

\begin{tabular}{|c|c|c|c|c|c|c|c|c|}
\hline \multirow{2}{*}{$\sum_{6}^{\stackrel{u}{u}}$} & 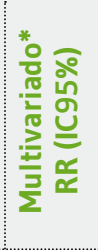 & 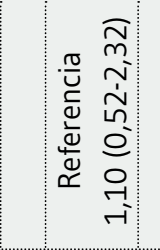 & 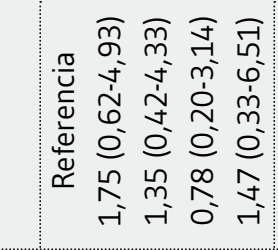 & & 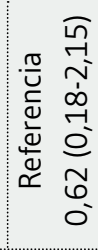 & & 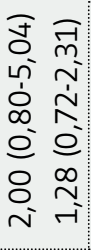 & 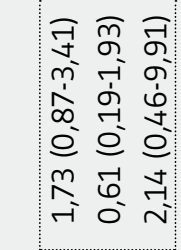 \\
\hline & 응 & 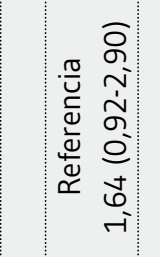 & 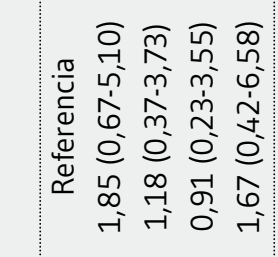 & & 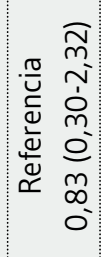 & & 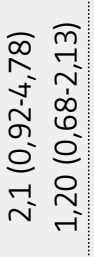 & 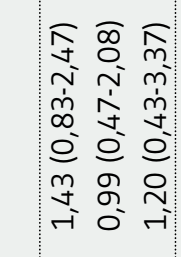 \\
\hline \multirow{2}{*}{ 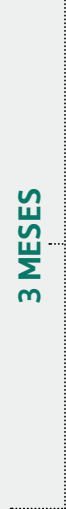 } & 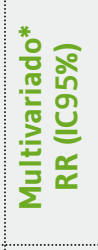 & 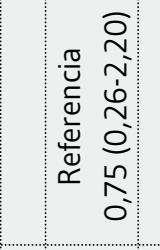 & 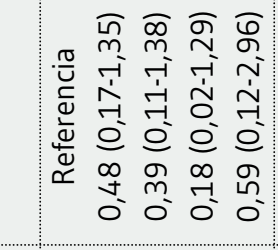 & & & & 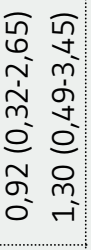 & 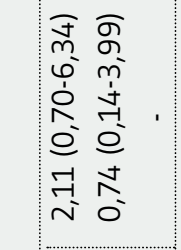 \\
\hline & 을 웡 & 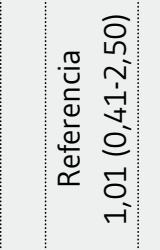 & 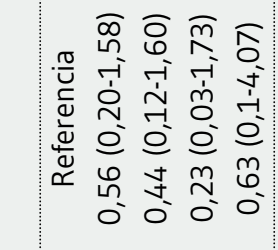 & & 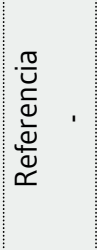 & & 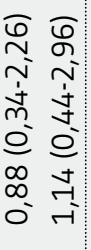 & 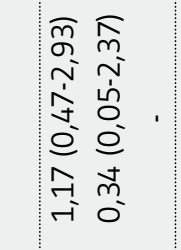 \\
\hline \multirow{2}{*}{$\underset{-}{\text { 崩 }}$} & 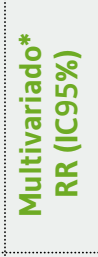 & 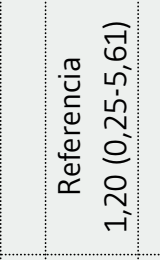 & 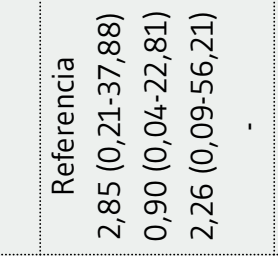 & & 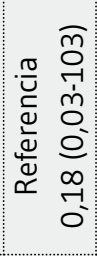 & & 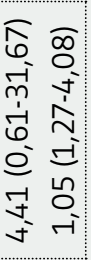 & 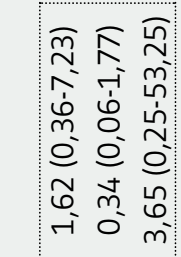 \\
\hline & 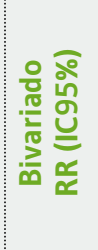 & 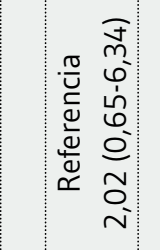 & 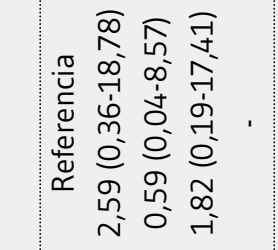 & & 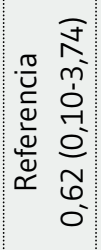 & & 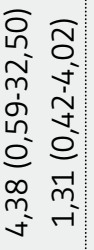 & 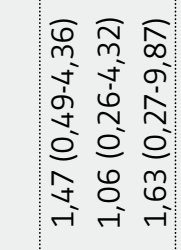 \\
\hline & & 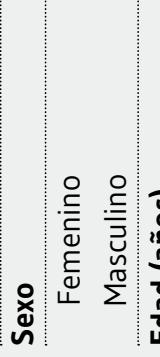 & 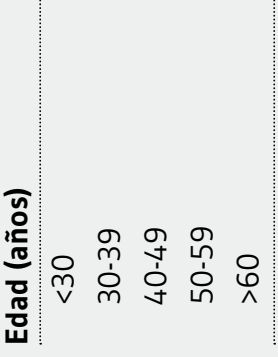 & 衣 & 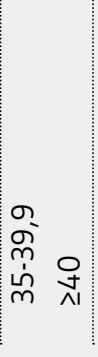 & 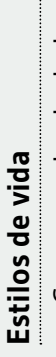 & 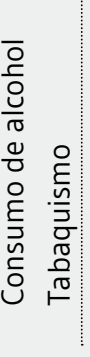 & 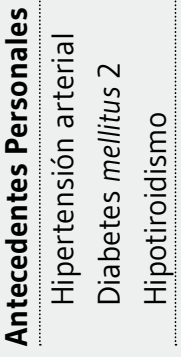 \\
\hline
\end{tabular}

IMC: Índice de Masa Corporal; RR: Riesgo Relativo; IC95\%: Intervalo de Confianza 95\%.

* Modelo presentado está ajustado por todas las variables listadas en la tabla. 
Tabla 5. Modelo crudo y ajustado: asociación de porcentaje de peso perdido y abandono del tratamiento nutricional.

\begin{tabular}{c|c|c|c|c} 
& \multicolumn{2}{|c|}{ Abandono al primer mes } & Modelo crudo & Modelo ajustado* \\
\hline Variación de peso & No n (\%) & Sí n (\%) & RR (IC95\%) & \\
\hline$<10 \%$ & $3(60,0)$ & $2(40,0)$ & Referencia & Referencia \\
$\geq 10 \%$ & $55(85,9)$ & $9(14,1)$ & $0,35(0,10-1,22)$ & $0,10(0,01-0,68)$ \\
& Abandono al tercer mes & Abandono al tercer mes & & \\
\hline Variación de peso & No & Sí & & \\
\hline$<10 \%$ & $3(60,0)$ & $2(40)$ & Referencia & Referencia \\
$\geq 10 \%$ & $51(79,7)$ & $13(20,3)$ & $0,50(0,16-1,66)$ & $0,27(0,06-1,10)$ \\
& Abandono al sexto mes & Abandono al sexto mes & & \\
\hline Variación de peso & No & Sín & & \\
\hline$<10 \%$ & $2(40,0)$ & $3(60)$ & Referencia & Referencia \\
$\geq 10 \%$ & $38(59,4)$ & $26(40,6)$ & $0,68(0,31-1,48)$ & $0,48(0,18-1,30)$ \\
\hline
\end{tabular}

n: muestra; RR: Riesgo Relativo; IC95\%: Intervalo de Confianza 95\%.

* Ajustado por edad y sexo.

en Inglaterra ${ }^{21}$. Por ello, debido a la transición nutricional en que los países de escasos recursos se encuentran, se necesita un protocolo adecuado para manejar a los pacientes bariátricos.

El 40\% de pacientes bariátricos abandonaron el tratamiento nutricional antes de iniciarlo y, de quienes asistieron a la primera cita, el 79,7\% no alcanzó los seis meses de seguimiento. Existen pocos estudios sobre el abandono de tratamiento nutricional en cirugía bariátrica, especialmente en países de escasos recursos. Por ejemplo, un programa canadiense para el manejo de peso reportó un $43 \%$ de abandono total, con una menor incidencia en pacientes que tuvieron cirugía bariátrica (12\%) comparado a los que fueron tratados con fármacos $(54 \%)^{13}$. Otro estudio tuvo una incidencia de abandono del $30 \%$ en pacientes bariátricos, pero una gran proporción de estos lo hicieron habiendo recibido asesoría nutricional personalizada ${ }^{22}$. Finalmente, una revisión a largo plazo de pérdida de peso después de una gastrectomía en manga laparoscópica incluyó 13 estudios y reportó una incidencia de abandono de $31 \%{ }^{23}$. Nuestro estudio expande el reporte de abandono al dividirlo en antes y después de recibir el tratamiento nutricional después de la cirugía bariátrica.

En adición, ninguna de las características de la población se asoció al abandono del tratamiento nutricional, lo cual podría ser causado por falta de potencia del estudio. Para el análisis de dicha asociación no se contó con la muestra total, ya que se perdieron participantes por no cumplir criterios de inclusión y por las altas tasas de abandono del tratamiento nutricional. Existe poca literatura acerca de la asociación entre los factores de riesgo y el abandono en cirugía bariátrica. Una revisión de ocho estudios sobre los factores de riesgo asociados a la atención posquirúrgica en cirugía bariátrica reportó pocos hallazgos consistentes: el peso antes de la cirugía y una mayor distancia de viaje fueron factores de riesgo de abandono ${ }^{24}$. Otro estudio determinó una menor edad e IMC como factores de riesgo para abandono de un tratamiento farmacológico en pacientes obesos ${ }^{13}$.

La literatura también señala factores de riesgo asociados a un tratamiento para la obesidad a largo plazo como ansiedad, estrés, insomnio y depresión severa ${ }^{18}$, así como resultados insatisfactorios y falta de motivación ${ }^{25}$, pero dichas variables no fueron evaluadas en este estudio.

A pesar de que la mayoría de los pacientes no llegó a cumplir los seis meses de tratamiento nutricional, se ha observado en otro estudio que la pérdida máxima de peso se da al año de la cirugía bariátrica restrictiva ${ }^{26}$. Independiente a la pérdida de peso, el paciente bariátrico debe continuar el tratamiento nutricional para mantener un estado óptimo de salud. Un seguimiento de cuatro años identifica deficiencias de micronutrientes como vitamina $D$, folato y vitamina $B_{12}$ en dichos pacientes ${ }^{27,28}$. En adición, se ha observado que la gastrectomía en manga induce a una pérdida importante de masa muscular y proteína sérica ${ }^{29}$. Por ende, se requiere adherencia al tratamiento nutricional para monitorear niveles de micronutrientes y proveer suplementación en caso necesario. Además, dicho tratamiento evitaría la reganancia de peso descrita a los 5 años poscirugía causado por una 
baja calidad de la dieta, sedentarismo y la falta de asesoramiento apropiado ${ }^{11,12}$.

El presente estudio multivariable muestra que aquellos pacientes que perdían más del $10 \%$ del peso en las primeras cinco semanas poscirugía tienen un riesgo menor de abandonar el tratamiento nutricional en el primer mes, pero no durante el tercero o sexto. Algunos estudios han reportado que las expectativas del paciente juegan un rol importante como factor de riesgo para abandonar. Por ejemplo, un estudio señala que el riesgo de abandonar incrementa por cada unidad que disminuya el IMC que se espera perder a los doce meses ${ }^{30}$. Además, el riesgo aumenta a los 6 meses de tratamiento ${ }^{30}$. En adición, otros estudios encontraron que, de manera general, los pacientes tienen expectativas poco realistas acerca de la pérdida de peso después de la cirugía bariátrica ${ }^{31,32}$. Los hallazgos del presente estudio confirman estos resultados: Ios participantes que perdían más del $10 \%$ del peso en las primeras cinco semanas tenían un menor riesgo de abandonar después de iniciar el tratamiento nutricional. A pesar de no ser significativos debido a problemas de tamaño de muestra, los resultados al tercer y sexto mes mostraron la misma tendencia. Esta asociación no ha sido reportada previamente por otros estudios.

Este puede ser el primer estudio en un país de Latinoamérica que muestra los criterios de inclusión de pacientes para cirugía bariátrica, así como las tasas de abandono del tratamiento nutricional en una muestra de pacientes sometidos a dicha cirugía. Asimismo, todos los pacientes fueron operados por un mismo cirujano utilizando la misma técnica y recibieron tratamiento nutricional por la misma nutricionista.

Por otro lado este estudio tiene varias limitaciones. Al ser retrospectivo, los datos a los que se pudo acceder fueron limitados, por ello no se pudo obtener información sobre predictores precoces de abandono que hubiera impactado los resultados de nuestro estudio, como: situación socioeconómica, nivel educativo, perfil psicológico, anamnesis alimentaria pre- y posquirúrgica. Igualmente, algunos datos antropométricos (perímetro abdominal) y bioquímicos (glicemia y perfil lipídico) que podrían haber dado una perspectiva médica adicional, no fueron registrados.

Otra limitación sería la falta de poder del estudio, especialmente para determinar asociaciones, ya que una mayor proporción de pacientes abandonó el tratamiento en los primeros meses del mismo. Otra posible limitación es haber utilizado una selección no probabilística de la muestra, ya que puede afectar su representatividad. Finalmente, podría existir un sesgo en la selección porque los pacientes pertenecían a una clínica privada y podrían no representar a otros centros, privados o públicos, pero muy pocos hospitales públicos realizan este tipo de cirugía en el Perú. Así, otros estudios, especialmente de carácter prospectivos son necesarios para evaluar predictores de abandono, los que permitirían identificar precozmente a aquellos pacientes con mayor riesgo de abandono al tratamiento nutricional.

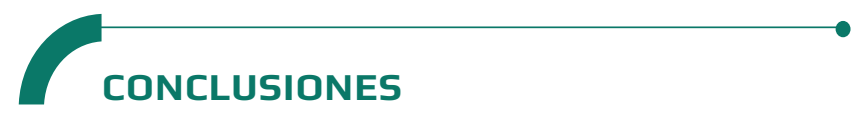

A pesar de que existen criterios internacionales para realizar la cirugía bariátrica, la gran mayoría de la población estudiada no los cumplen. Las tasas de abandono son altas tanto antes como después del tratamiento nutricional. Además, una pérdida de peso $\geq 10 \%$ en las primeras cinco semanas se asocia a un menor abandono del tratamiento nutricional en el primer mes, pero no en el tercero o sexto. Los resultados resaltan la urgencia de disponer de un protocolo apropiado para el manejo de cirugía bariátrica en el contexto peruano, que especifique las indicaciones de la misma siguiendo lo establecido internacionalmente, así como definir apropiadamente el equipo multidisciplinario pre- y posquirúrgico, y el rol de cada uno de los miembros de este equipo, especialmente en lo relacionado al tratamiento nutricional antes y después de la cirugía.

\section{CONFLICTO DE INTERESES}

Los autores expresan que no hay conflictos de interés al redactar el manuscrito.

\section{REFERENCIAS}

(1) NCD Risk Factor Collaboration (NCD-RisC). Trends in adult body-mass index in 200 countries from 1975 to 2014: a pooled analysis of 1698 population-based measurement studies with 19.2 million participants. Lancet. 2016; 387(10026): 1377-96.

(2) Álvarez-Dongo D, Sánchez-Abanto J, Gómez-Guizado G, TarquiMamani C. Sobrepeso y obesidad: prevalencia y determinantes sociales del exceso de peso en la población peruana (20092010). Rev Peru Med Exp Salud Publica. 2012; 29(3): 303-13.

(3) Kelly T, Yang W, Chen C-S, Reynolds K, He J. Global burden of obesity in 2005 and projections to 2030. Int J Obes. 2008; 32(9): 1431-7.

(4) Sjöström L, Lindroos A-K, Peltonen M, Torgerson J, Bouchard C, Carlsson B, et al. Lifestyle, diabetes, and cardiovascular risk factors 10 years after bariatric surgery. N Engl ] Med. 2004; 351(26): 2683-93. 
(5) Borisenko O, Colpan Z, Dillemans B, Funch-Jensen P, Hedenbro J, Ahmed AR. Clinical Indications, Utilization, and Funding of Bariatric Surgery in Europe. Obes Surg. 2015; 25(8): 1408-16.

(6) Fried M, Yumuk V, Oppert J-M, Scopinaro N, Torres AJ, Weiner $R$, et al. Interdisciplinary European Guidelines on metabolic and bariatric surgery. Obes Facts. 2013; 6(5): 449-68.

(7) Mechanick JI, Youdim A, Jones DB, Garvey WT, Hurley DL, McMahon MM, et al. Clinical practice guidelines for the perioperative nutritional, metabolic, and nonsurgical support of the bariatric surgery patient-2013 update: cosponsored by American Association of Clinical Endocrinologists, The Obesity Society, and American Society for Metabolic \& Bariatric Surgery. Obesity. 2013; 21(Suppl 1): S1-27.

(8) Faria SL, de Oliveira Kelly E, Lins RD, Faria OP. Nutritional management of weight regain after bariatric surgery. Obes Surg. 2010; 20(2): 135-9.

(9) Herrera M, Alvar I. Protocolo Referencia y Contrarreferencia Procedimiento "Cirugía Bariátrica" en el Programa de Tratamiento Integral de la Obesidad. Servicio de Salud de Osorno, Chile; 2013.

(10) Vojvodic I. Cuestionamientos a la cirugía bariátrica. Rev Gastroenterol Peru. 2009; 29(4): 355-61.

(11) Bastos ECL, Barbosa EMWG, Soriano GMS, dos Santos EA, Vasconcelos SML. Determinants of weight regain after bariatric surgery. Arq Bras Cir Dig. 2013; 26(Suppl 1): 26-32.

(12) Freire RH, Borges MC, Alvarez-Leite Jl, Toulson Ml. Food quality, physical activity, and nutritional follow-up as determinant of weight regain after Roux-en-Y gastric bypass. Nutrition. 2012; 28(1): 53-8.

(13) Gill RS, Karmali S, Hadi G, Al-Adra DP, Shi X, Birch DW. Predictors of attrition in a multidisciplinary adult weight management clinic. Can J Surg. 2012; 55(4): 239-43.

(14) Handjieva-Darlenska T, Holst C, Grau K, Blaak E, Martinez JA, Oppert J-M, et al. Clinical correlates of weight loss and attrition during a 10-week dietary intervention study: results from the NUGENOB project. Obes Facts. 2012; 5(6): 928-36.

(15) Lent MR, Eichen DM, Goldbacher E, Wadden TA, Foster GD. Relationship of food addiction to weight loss and attrition during obesity treatment. Obesity (Silver Spring). 2014; 22(1): 52-5.

(16) Crawford R, Glover L. The impact of pre-treatment weight-loss expectations on weight loss, weight regain, and attrition in people who are overweight and obese: A systematic review of the literature. $\mathrm{Br}]$ Health Psychol. 2012; 17(3): 609-30.

(17) Gómez C, Palma S, Piedra M, Bermejo L, Loria V. Eficacia y predictores de cumplimiento de un programa tarapéutico en pacientes con obesidad grado II complicada o mórbida, no candidatos a programa de cirugía. Nutr Clin Diet Hosp. 2009; 29(3): 25-31.

(18) Michelini I, Falchi AG, Muggia C, Grecchi I, Montagna E, De Silvestri $A$, et al. Early dropout predictive factors in obesity treatment. Nutr Res Pract. 2014; 8(1): 94-102.
(19) Hatoum IJ, Kaplan LM. Advantages of percent weight loss as a method of reporting weight loss after Roux-en-Y gastric bypass. Obesity. 2013; 21(8): 1519-25.

(20) Bucknor A, Ekwobi C. Need for guidelines on body recontouring after bariatric surgery. BM]. 2014; 349: g7637.

(21) Ahmad A, Laverty AA, Aasheim E, Majeed A, Millett C, Saxena S. Eligibility for bariatric surgery among adults in England: analysis of a national cross-sectional survey. JRSM Open. 2014; 5(1): 2042533313512479.

(22) Diamant A, Milner J, Cleghorn M, Sockalingam S, Okrainec A, Jackson TD, et al. Analysis of patient attrition in a publicly funded bariatric surgery program. ] Am Coll Surg. 2014; 219(5): 1047-55.

(23) Diamantis T, Apostolou KG, Alexandrou A, Griniatsos J, Felekouras $E$, Tsigris C. Review of long-term weight loss results after laparoscopic sleeve gastrectomy. Surg Obes Relat Dis. 2014; 10(1): 177-83.

(24) Moroshko I, Brennan L, O'Brien P. Predictors of attrition in bariatric aftercare: a systematic review of the literature. Obes Surg. 2012; 22(10): 1640-7.

(25) Dalle Grave R, Melchionda N, Calugi S, Centis E, Tufano A, Fatati $\mathrm{G}$, et al. Continuous care in the treatment of obesity: an observational multicentre study. J Intern Med. 2005; 258(3): 265-73.

(26) Courcoulas AP, Christian NJ, Belle SH, Berk PD, Flum DR, Garcia $L$, et al. Weight change and health outcomes at 3 years after bariatric surgery among individuals with severe obesity. JAMA. 2013; 310(22): 2416-25.

(27) Alexandrou A, Armeni E, Kouskouni E, Tsoka E, Diamantis T, Lambrinoudaki I. Cross-sectional long-term micronutrient deficiencies after sleeve gastrectomy versus Roux-en-Y gastric bypass: a pilot study. Surg Obes Relat Dis. 2014; 10(2): 262-8.

(28) Handzlik-Orlik G, Holecki M, Orlik B, Wyleżoł M, Duława J. Nutrition management of the post-bariatric surgery patient. Nutr Clin Pract. 2015; 30(3): 383-92.

(29) Friedrich AE, Damms-Machado A, Meile T, Scheuing N, Stingel K, Basrai M, et al. Laparoscopic sleeve gastrectomy compared to a multidisciplinary weight loss program for obesity-effects on body composition and protein status. Obes Surg. 2013; 23(12): 1957-65.

(30) Dalle Grave R, Calugi S, Molinari E, Petroni ML, Bondi M, Compare $\mathrm{A}$, et al. Weight loss expectations in obese patients and treatment attrition: an observational multicenter study. Obes Res. 2005; 13(11): 1961-9.

(31) Kaly P, Orellana S, Torrella T, Takagishi C, Saff-Koche L, Murr MM. Unrealistic weight loss expectations in candidates for bariatric surgery. Surg Obes Relat Dis. 2008; 4(1): 6-10.

(32) Price HI, Gregory DM, Twells LK. Weight loss expectations of laparoscopic sleeve gastrectomy candidates compared to clinically expected weight loss outcomes 1-year post-surgery. Obes Surg. 2013; 23(12): 1987-93. 